OPEN ACCESS

Edited by:

Tao Yang,

Shanghai Jiao Tong University, China

Reviewed by:

Jie Tang,

Southern Medical University, China

Wenwen Liu,

Shandong University, China

*Correspondence:

Yu Sun

sunyu@hust.edu.cn

†These authors have contributed equally to this work

Specialty section

This article was submitted to

Molecular Medicine,

a section of the journal

Frontiers in Cell and Developmental

Biology

Received: 27 January 2021

Accepted: 19 February 2021

Published: 18 March 2021

Citation:

Bai X, Chen S, Xu K, Jin Y, Niu X, Xie L, Qiu Y, Liu X-Z and Sun Y (2021)

$\mathrm{N}$-Acetylcysteine Combined With

Dexamethasone Treatment Improves

Sudden Sensorineural Hearing Loss and Attenuates Hair Cell Death

Caused by ROS Stress.

Front. Cell Dev. Biol. 9:659486. doi: 10.3389/fcell.2021.659486

\section{N-Acetylcysteine Combined With Dexamethasone Treatment Improves Sudden Sensorineural Hearing Loss and Attenuates Hair Cell Death Caused by ROS Stress}

\author{
Xue Bait, Sen Chent, Kai Xut, Yuan Jin, Xun Niu, Le Xie, Yue Qiu, Xiao-Zhou Liu and \\ Yu Sun* \\ Department of Otorhinolaryngology, Union Hospital, Tongji Medical College, Huazhong University of Science \\ and Technology, Wuhan, China
}

Sudden sensorineural hearing loss (SSNHL) is a common emergency in the world. Increasing evidence of imbalance of oxidant-antioxidant were found in SSNHL patients. Steroids combined with antioxidants may be a potential strategy for the treatment of SSNHL. In cochlear explant experiment, we found that $\mathrm{N}$-acetylcysteine (NAC) combined with dexamethasone can effectively protect hair cells from oxidative stress when they were both at ineffective concentrations alone. A clinic trial was designed to explore whether oral NAC combined with intratympanic dexamethasone (ITD) as a salvage treatment has a better therapeutic effect. 41 patients with SSNHL were randomized to two groups. 23 patients in control group received ITD therapy alone, while 18 patient $s$ in NAC group were treated with oral NAC and ITD. The patients were followed-up on day 1st (initiation of treatment) and day 14th. Overall, there was no statistical difference in final pure-tone threshold average (PTA) improvement between those two groups. However, a significant hearing gain at $8,000 \mathrm{~Hz}$ was observed in NAC group. Moreover, the hearing recovery rates of NAC group is much higher than that in control group. These results demonstrated that oral NAC in combination with ITD therapy is a more effective therapy for SSNHL than ITD alone.

Keywords: sudden sensorineural hearing loss, hair cell, ROS, N-acetylcysteine, steroid

\section{INTRODUCTION}

Sudden sensorineural hearing loss (SSNHL) is considered one of the most common emergencies in clinical practice. In the United States, SSNHL is thought to affect between 5-27 in 100,000 individuals, with about 66,000 new cases per year (Alexander and Harris, 2013; Chandrasekhar et al., 2019). In Japan, there are 60.9 cases per 100,000 population diagnosed with SSNHL annually (Nakashima et al., 2014). Further epidemiological investigations have shown that the incidence of SSNHL is increasing globally (Michel, 2011; Kitoh et al., 2020). Currently, high-dose systemic steroid treatment is used as the first-line treatment of SSNHL (Chandrasekhar et al., 2019; 
Kitoh et al., 2020). However, approximately 50\% of patients experience no or limited hearing improvement after systemic steroid treatment (Hunchaisri et al., 2010; Tong et al., 2020). Therefore, those patients with limited hearing improvement (less than 10-20 dB) are considered to have refractory sudden hearing loss (RSHL) (Hunchaisri et al., 2010; Ferri et al., 2012). Although intratympanic dexamethasone (ITD) therapy has been recommended as a salvage treatment for RSHL or after failure of systemic steroid treatment (Moon et al., 2011; Berjis et al., 2016; Sun et al., 2018), its efficacy remains unsatisfactory (Li et al., 2015). It is therefore necessary to devise new strategies for SSNHL.

Steroids combined with another therapy is a common strategy for the treatment of SSNHL. In the United States, hyperbaric oxygen therapy (HBOT) combined with ITD is one option for salvage therapy (Chandrasekhar et al., 2019). Meanwhile, prostaglandin E1 combined with steroids has been recommended by Japanese clinicians for severe to profound SSNHL (Kitoh et al., 2020). Pharmacologically, combination therapy has unique advantages, with potential synergistic effects to achieve better therapeutic outcomes. Recently, antioxidants have been removed from the list of interventions that the American Clinical Practice Guidelines for SSNHL (published in 2019) recommend against using (Chandrasekhar et al., 2019). Although no explanation is given for this change, it indicates that antioxidants may have potential value in the treatment of SSNHL.

To date, a wide variety of antioxidants have been used in the treatment of SSNHL, but their effects remain controversial. Previous studies showed that different combinations or single vitamins (used as antioxidants, vitamin $\mathrm{A}, \mathrm{C}$, or $\mathrm{E}$ ) combined with a steroid were more beneficial for patients with SSNHL (Joachims et al., 2003; Hatano et al., 2008; Kang et al., 2013; Kaya et al., 2015). Similarly, a clinical trial showed that a zinc supplement may enhance the hearing recovery of SSNHL patients by reducing oxidative stress of the cochlea (Yang et al., 2011). However, another study did not find any convincing benefits of a zinc supplement (Niran et al., 2015). Although evidence of an oxidant-antioxidant imbalance was found in SSNHL patients, the therapeutic targets of antioxidants and the mechanism of their interaction with steroids are still difficult to fully elucidate (Jarosław et al., 2019; Ozdamar et al., 2019). Therefore, how to select the effective antioxidant for SSNHL has become a puzzled problem to be solved.

$\mathrm{N}$-acetylcysteine (NAC), as a precursor of glutathione (GSH) and a limiting factor in the process of GSH synthesis, is one of the antioxidants commonly used in the inner ear (Duan et al., 2004; Pathak et al., 2015; Tillinger et al., 2018). It has been clinically proven to be effective as a single therapy in the treatment of SSNHL or cisplatin-induced hearing loss (Riga et al., 2013; Chen and Young, 2016). For initial treatment, combination therapy with corticosteroids plus L-NAC is associated with improved hearing compared to corticosteroids alone (Angeli et al., 2012). Moreover, addition of NAC has been shown to increase glucocorticoid sensitivity in a mouse model of steroidresistant asthma (Eftekhari et al., 2013). These studies indicate that NAC and steroids may enhance treatment efficacy through synergistic action. Combining a steroid with NAC may be a potential alternative to salvage therapy of SSNHL or RSHL. To prove our hypothesis, in vitro experiments were performed to verify whether NAC and steroid have a synergistic effect on oxidative stress injury. In addition, a clinical trial was designed to compare the therapeutic efficacy of ITD with that of ITD combined with NAC in the salvage therapy of SSNHL.

\section{MATERIALS AND METHODS}

\section{Culture of Cochlear Explants and Drug Treatments}

C57BL/6 mice at P3 were decapitated after anesthesia, then the cochlear basilar membrane was carefully isolated from the cochlea in transparent Hank's balanced salt solution (PB180321, ProCell, Wuhan, China). The cochlear basilar membrane containing the organ of Corti was transferred onto a collagen gel matrix. A $15 \mu \mathrm{L}$ droplet of a 9:1:1 rat tail collagen (Type 1-4236, BD Biosciences, Franklin Lakes, NJ, United States), $10 \times$ Basal Medium Eagle (BME; B9638, Sigma-Aldrich, St. Louis, MO, United States), 2\% sodium carbonate (P1110, Solarbio, Beijing, China) mixture was placed on the surface of a $35-\mathrm{mm}$ culture dish and allowed to gel for approximately $30 \mathrm{~min}$ at $37^{\circ} \mathrm{C}$. Afterward, $1.3 \mathrm{~mL}$ medium consisting of $1 \times$ BME (41010109, Gibco, Carlsbad, CA, United States) containing 1\% bovine serum albumin (A8020, Solarbio), 10\% glutamine (G7513, SigmaAldrich), $5 \mathrm{mg} / \mathrm{mL}$ glucose and 10,000 U/mL penicillin $\mathrm{G}$ (P3414, Sigma-Aldrich) were added to the culture dish. The cochlear explants were placed as a flat preparation on the surface of the collagen gel, and the surface of the basilar membrane was exactly even with the culture medium. All explants were incubated overnight at $37^{\circ} \mathrm{C}$ in an atmosphere of $5 \% \mathrm{CO}_{2}$. On the following day, the culture medium was removed, the explants of the cochlea for primary culture were treated with fresh medium containing drugs for $24 \mathrm{~h}$ in vitro, then subjected to immunofluorescent staining. The cochlear explants were divided into four groups and were exposed to $160 \mathrm{U} / \mathrm{L}$ glucose oxidase (GO; G3660, Sigma-Aldrich; GO group), $160 \mathrm{U} / \mathrm{L}$ GO together with $50 \mu \mathrm{g} / \mathrm{mL}$ dexamethasone (GO + Dex group), $160 \mathrm{U} / \mathrm{L}$ GO with $5 \mathrm{mM}$ NAC (A7250, Sigma-Aldrich; GO + NAC group), or $160 \mathrm{U} / \mathrm{L}$ GO together with $5 \mathrm{mM} \mathrm{NAC}$ and $50 \mu \mathrm{g} / \mathrm{mL}$ dexamethasone (GO + Dex + NAC group). The cochlear explants $(n=3-5$ in each group) were incubated at $37^{\circ} \mathrm{C}$ in $5 \% \mathrm{CO}_{2}$ for $24 \mathrm{~h}$ and then harvested for further experiments.

\section{Cochlear Tissue Preparation and Fluorescent Labeling}

The cochlear explants were fixed in $4 \%$ paraformaldehyde in $0.01 \mathrm{M}$ PBS for $1 \mathrm{~h}$ at room temperature. After washing three times in $0.01 \mathrm{M}$ PBS, explants were stained with DAPI (C1005, Beyotime Institute of Biotechnology, Jiangsu, China) and phalloidin (0.05 mg/mL, P5282, Sigma-Aldrich) for $10 \mathrm{~min}$ each. Images were captured with a laser scanning confocal microscope (Nikon, Tokyo, Japan). Three regions from the apical, middle, and basal turns of the stretched cochlear explants were scanned using a $\times 60$ magnification lens. 


\section{Clinical Study Design and Patients}

This clinical trial was carried out between March 2017 and March 2019 at the Department of Otorhinolaryngology of Wuhan Union hospital. Eligible subjects were patients with at least $30 \mathrm{~dB}$ hearing loss in three contiguous frequencies that had occurred over a course of 3 days, with available previous audiometry data. All patients had a normal otoscopic exam and tympanograms and had not responded to initial treatment. The hearing thresholds of patients were measured at 250-8,000 Hz. Exclusion criteria for the study were: Subjects older than 60 years old (to rule out potential presbycusis); patients with completely hearing loss at 4,000 and $8,000 \mathrm{~Hz}$; patients with Meniere's disease or other recognized pathologies of SSHL, such as genetic causes, acoustic trauma, previous otologic surgery and so on; any contraindication for the use of NAC and steroids, such as pregnancy or hypertension; MRI scan finding acoustic neuroma or other retrocochlear lesions; disease onset time of more than 14 days; incomplete medical records or inadequate follow-up. All individuals underwent medical history, physical examinations, and laboratory tests, as well as audiologic evaluations that included tympanometry and pure tone audiometry before diagnosis and therapy. All individuals were informed about the procedure and the possible risks. They all agreed to participate in this research and signed an informed consent form. This study was approved by the institutional review board of Wuhan Union hospital.

\section{Therapy Protocol}

After screening for eligibility, all subjects were randomly divided into two groups. Randomization was carried out by generating sequential random numbers using computer-based software. Every recruited individual received sequential random numbers placed in closed envelopes. All doctors and patients were aware of the allocation. The physicians that performed the pure tone audiometry and data analysis were kept blinded to the allocation.

All of the eligible subjects had received ITD injections and basic treatment for SSNHL, which included nourishing nerves and improving vascular microcirculation. All patients underwent hearing tests before treatment and 2 weeks later after treatment. The patients were treated by the senior physicians and received ITD administration alone in the control group. In the experimental groups (NAC groups), all patients routinely received combination therapy with ITD plus oral NAC. NAC (Conbe Biopharmaceutical Company, China) was given orally in the form of effervescent tablets at a dose of $600 \mathrm{mg}$ two times daily for 2 weeks, starting with the with the first IT Dex therapy.

\section{Measurement of Auditory Function}

The audiometric data of all evaluable patients were analyzed. The pure-tone hearing thresholds were measured at 250, 500, $1,000,2,000,4,000$, and $8,000 \mathrm{~Hz}$. The pure-tone threshold average (PTA) was calculated by measuring the six-frequency average of the threshold value at 500, 1,000, 2,000, and 4,000 $\mathrm{Hz}$. Thresholds that were not measurable because of the limit of the audiometric equipment were coded with the maximum level of the audiometer that was set at $120 \mathrm{~dB}$ (HL). Pure tone audiometry was performed before initiation of treatment and 2 weeks after initiation of treatment. The main end-point of this research was the final mean hearing improvement, which was regarded as the difference between initial and final PTA. PTA values were compared to assess the hearing recovery before and after treatment. According to the criteria in the guidelines for the diagnosis and treatment of sudden deafness of the Chinese society of otorhinolaryngology, "hearing improvement" was defined as more than $15 \mathrm{~dB}$ hearing gain, and "no improvement" as less than $15 \mathrm{~dB}$ hearing gain.

\section{Cell Culture and Treatment}

BxPC3 cells were cultured in high-glucose DMEM (11995500, Gibco) mixed with 5\% volume of fetal bovine serum (11054001, Gibco) with antibiotics and incubated in $5 \% \mathrm{CO}_{2}$ at $37^{\circ} \mathrm{C}$. The levels of ROS in cells were detected by staining with dichlorodihydrofluorescein diacetate (DCFH-DA; D6883, Sigma-Aldrich). The cells were exposed to $80 \mathrm{U} / \mathrm{L}$ glucose oxidase (GO, GO group) for $4 \mathrm{~h}$. Cells after treatment were washed in pre-warmed PBS and stained with $10 \mu \mathrm{M}$ DCFH-DA in serum-free DMEM for $30 \mathrm{~min}$. The cell fluorescence intensity was measured by fluorescence microscopy.

\section{Statistical Analysis}

Efficacy was analyzed in all eligible patients. Descriptive statistics were used for the feature description. Paired samples $t$ tests were used to compare the means of quantitative variables in the same group at different points in time. Independent samples $t$ tests were used to compare the means of metric variables between two groups. Categorial variables were compared using Fisher's exact test or Chi-square test. A difference was considered to be statistically significant when the $P$ value was less than 0.05 . All statistical analyses were performed using the SPSS statistical software package (version 22.0; IBM SPSS Statistics for Windows, Armonk, NY, United States). The graphs were created using GraphPad Prism (version 8.2.1).

\section{RESULTS}

\section{A Cochlear Explant Model of Oxidative Stress Injury Was Established to Verify the Oto-Protective Synergistic Effect of Dexamethasone Combined With NAC}

In patients with SSNHL, some indirect evidence of oxidative stress injury has been found successively (Becatti et al., 2017; Jarosław et al., 2019). Therefore, a cochlear explant model of oxidative stress injury was adopted to explore the effects of combined therapy. In the BxPC3 cell line, $4 \mathrm{~h}$ of $\mathrm{GO}$ treatment significantly increased the intracellular ROS level. The green fluorescent signal in the GO group detected by DCFHDA, a probe of reactive oxygen species, was much stronger than that of the control group (Figures 1A-D). Therefore, GO was used to increase the ROS level in cochlear explants. Compared to the control group (Figures $\mathbf{1 E - G}, n=3$ ), a 


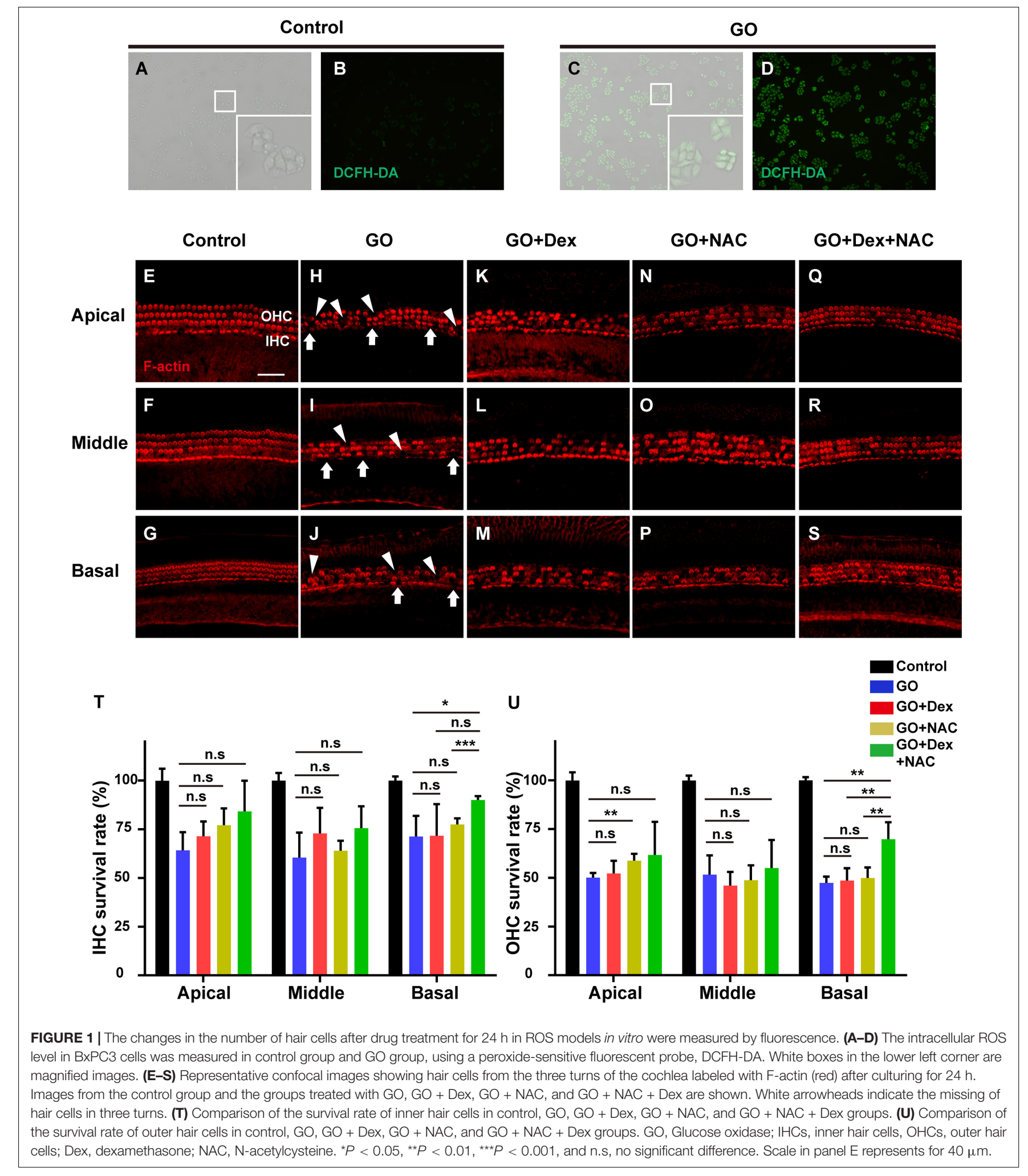

moderate degeneration of hair cells was observed in different turns of the GO group $(n=4)$. After $24 \mathrm{~h}$ of GO incubation, half of the outer hair cells (OHCs) had died, while 60.51$71.34 \%$ of inner hair cells (IHCs) survived (hair cell loss, white arrows and arrowheads, Figures $\mathbf{1 H}-\mathbf{J})$. A low concentration of dexamethasone $(50 \mu \mathrm{g} / \mathrm{mL})$ was added to GO-treated explants $(\mathrm{GO}+$ Dex group), and the results revealed that dexamethasone had no protective effect on hair cells at this concentration. The 
rates of $\mathrm{OHC}$ survival were $52.25 \pm 6.46,46.09 \pm 6.97$, and $48.64 \pm 6.32 \%$ in the apical, middle and basal turns, respectively (GO + Dex group, $n=5$ ). Meanwhile, approximately $70 \%$ of IHC survived in the GO + Dex group (Figures $\mathbf{1 K}-\mathbf{M}$ ). Similarly, a non-therapeutic concentration of NAC (5 mM) was also added to the GO-treated explant (GO + NAC group). In this group, the average $\mathrm{OHC}$ survival rates were $48.87-58.80 \%$ in different turns, while IHC survival rates fluctuated between 64.09 and $77.56 \%$ (Figures 1N-P). However, there was a statisticallysignificant difference in the $\mathrm{OHC}$ survival rate of the apical turn between the GO and GO + NAC groups (GO: $50.16 \pm 2.36 \%$ vs. GO + NAC: $58.50 \pm 3.54 \%, P=0.0042)$. This improvement of OHC survival was fairly limited (less than 9\%). Except for the above improvement, there was no statistically-significant difference in hair cell survival rates between the GO + NAC group and the GO group (Figures 1T,U). When Dex and NAC were both added to GO-treated cochlear explants $(n=5)$, the number of surviving IHCs or OHCs in the basal turn was significantly increased (Figures 1Q-S). Compared with the GO group, the survival rates of $\mathrm{OHC}$ and IHC in the basal turn of the GO + Dex + NAC group were significantly increased $(\mathrm{OHC}$, $47.41 \pm 3.20$ vs. $69.85 \pm 8.65 \%, P=0.0018$; IHC, $71.34 \pm 10.56$ vs. $90.00 \pm 2.00 \%, P=0.0057$, Figures 1 T,U).

\section{Clinical Trial to Observe the Effectiveness of NAC Combined With Dexamethasone Therapy}

There was a total of 64 patients who agreed to take part in this study. Of those, 14 were excluded for not conforming to eligibility criteria, consisting of three patients who had experienced symptoms for more than 14 days, one who had uncontrolled diabetes mellitus, one patient with a history of Meniere's disease, two patients who had not undergone any initial treatment at other hospitals, four patients who did not have hearing loss in five frequencies, and three participants who declined to take part. The remaining 50 patients who agreed to participate were randomized into two groups for further treatment and analysis. Of the 50 participants included, five were later excluded because of loss of contact from this clinical trial, and four patients were excluded owing to withdrawal of consent. Finally, overall 41 patients were included in our analysis. There were 23 patients in the control group, while 18 patients were analyzed in the NAC group (Figure 2).

All 41 patients were recruited at the Department of Otorhinolaryngology of Wuhan Union hospital. Sixteen of the patients were male $(39.0 \%)$ and 25 were female $(61.0 \%)$ with an average age of $38.5 \pm 14.4$ years (range: 14-60). Twenty-three (56.1\%) participants were randomized to the control group, and $18(43.9 \%)$ to the NAC group. The initial PTA of all patients was $64.1 \pm 21.5 \mathrm{~dB}$ HL $(66.25 \pm 20.17 \mathrm{~dB}$ in the control group, $61.3 \pm 22.8 \mathrm{~dB}$ in the NAC group). The post-treatment PTA of the control group was $54.7 \pm 25.4 \mathrm{~dB} \mathrm{HL}$, while it was $42.2 \pm 26.3 \mathrm{~dB}$ $\mathrm{HL}$ in the NAC group. For all patients, the mean duration of hospital treatment was $11.8 \pm 4.7$ days $(11.8 \pm 4.9$ days in the control group; $12 \pm 4.5$ days in the NAC group). The mean PTA

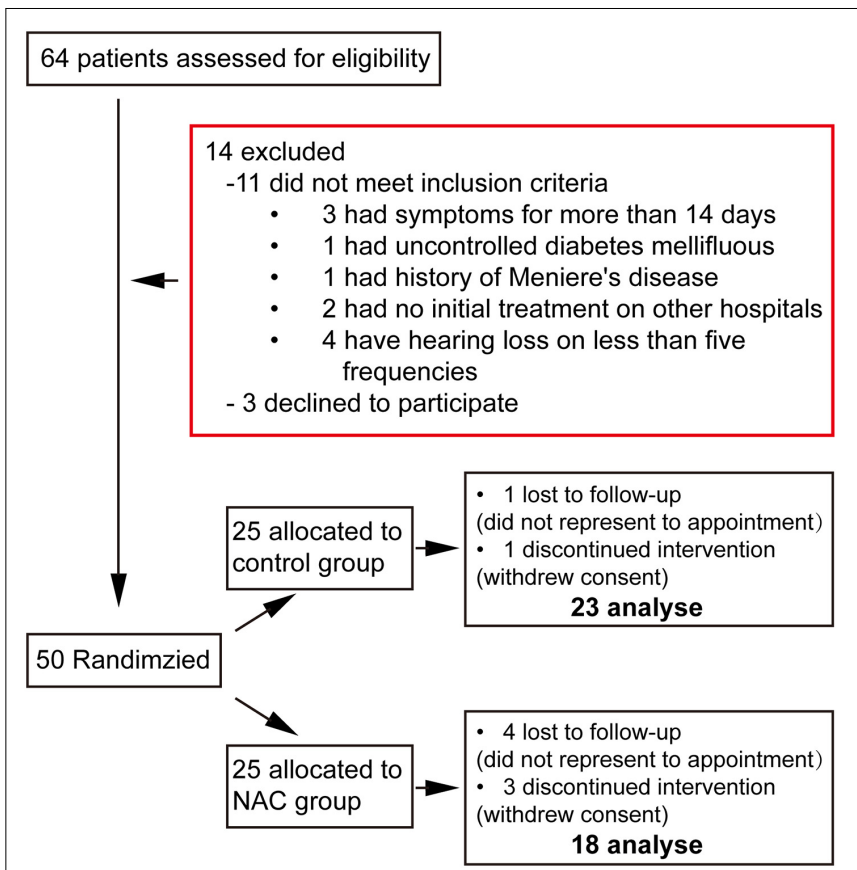

FIGURE 2 | Study flowchart.

gain of all patients was $14.9 \pm 15.5 \mathrm{~dB}(11.6 \pm 17.8 \mathrm{~dB}$ in the control group; $19.0 \pm 11.1 \mathrm{~dB}$ in the NAC group). No statisticallysignificant differences were found between the control and NAC groups concerning age, sex, days in hospital, initial PTA, final PTA, or mean PTA gain $(P>0.05$, Table 1$)$.

Figure 3 indicates the audiologic outcomes at different frequencies for all patients in the control and NAC groups. The hearing gains of the control group were 17.2 $\pm 18.5,17.2 \pm 20.7$, $12.8 \pm 16.1,8.9 \pm 17.6,7.4 \pm 24.1$, and $6.1 \pm 22.1 \mathrm{~dB}$ at 250, $500,1,000,2,0004,000$, and $8,000 \mathrm{~Hz}$, respectively. These gains in the NAC group were $15.6 \pm 18.5,18.3 \pm 13.7,20.3 \pm 10.8$, $17.2 \pm 12.6,20.3 \pm 16.6$, and $20.8 \pm 14.8 \mathrm{~dB}$, respectively, at the corresponding frequencies. Compared with the control group, the mean gain of the NAC group was significantly different at $8,000 \mathrm{~Hz}(P=0.019$, Power $=0.854$, Figure 3G). No statistically-significant differences were detected at any of the other frequencies $(P>0.05$, Figures 3A-F).

In terms of hearing recovery (Table 2), any PTA gain $(500-4,000 \mathrm{~Hz})$ greater than $15 \mathrm{~dB}$ is considered effective. The percentage of patients who experienced effective recovery in the control group vs. the NAC group was $39.1 \%$ (9 of 23 ) vs. $72.2 \%$ (13 of 18). Thus there was a significant difference in the effective rate between control and NAC groups $(P=0.035$, Pearson's chi-squared test).

\section{DISCUSSION}

Hair cells mainly function in transducing sound waves into the electric signals (Wang Y. et al., 2017; Liu Y. et al., 2019; Qi et al., 2019). Hearing loss could be caused by genetic factors, 
TABLE 1 | Demographic and audiological features of patients in control group and NAC group.

\begin{tabular}{lccr}
\hline & All $(\boldsymbol{n}=\mathbf{4 1})$ & Control $(\boldsymbol{n}=\mathbf{2 3})$ & NAC $(\boldsymbol{n}=\mathbf{1 8})$ \\
\hline Age (years) (mean \pm SD) & $38.54 \pm 14.35$ & $41.96 \pm 12.50$ & $34.17 \pm 15.69$ \\
Gender (male/female) & $16: 25$ & $8: 15$ & $8: 10$ \\
Time in hospital (days) (mean \pm SD) & $11.88 \pm 4.66$ & $11.78 \pm 4.88$ & $12.00 \pm 4.50$ \\
Initial PTA (dB) (mean \pm SD) & $64.05 \pm 21.52$ & $66.25 \pm 20.72$ & $61.25 \pm 22.78$ \\
Final PTA (dB) (mean \pm SD) & $49.21 \pm 26.20$ & $54.67 \pm 25.37$ & $42.22 \pm 26.26$ \\
Mean PTA gain (Db) (mean \pm SD) & $14.85 \pm 15.54$ & $11.57 \pm 17.84$ & $19.03 \pm 11.09$ \\
ALT (U/T) (mean \pm SD) & $23.63 \pm 16.08$ & $23.52 \pm 14.41$ & $23.78 \pm 18.43$ \\
Blood fat (Dyslipidemia/Ortholiposis) & $19: 22$ & $10: 13$ & $9: 9$ \\
NLR (mean \pm SD) & $2.22 \pm 1.16$ & $2.12 \pm 0.74$ & 0.46 \\
\hline
\end{tabular}

PTA, pure tone audiometry; ALT, alanine transaminase; NLR, neutrophil-to-lymphocyte ratio; SD, standard deviation; NAC, N-acetylcysteine. PTA pure tone average at 500, 1,000, 2,000, and 4,000 Hz hearing thresholds.

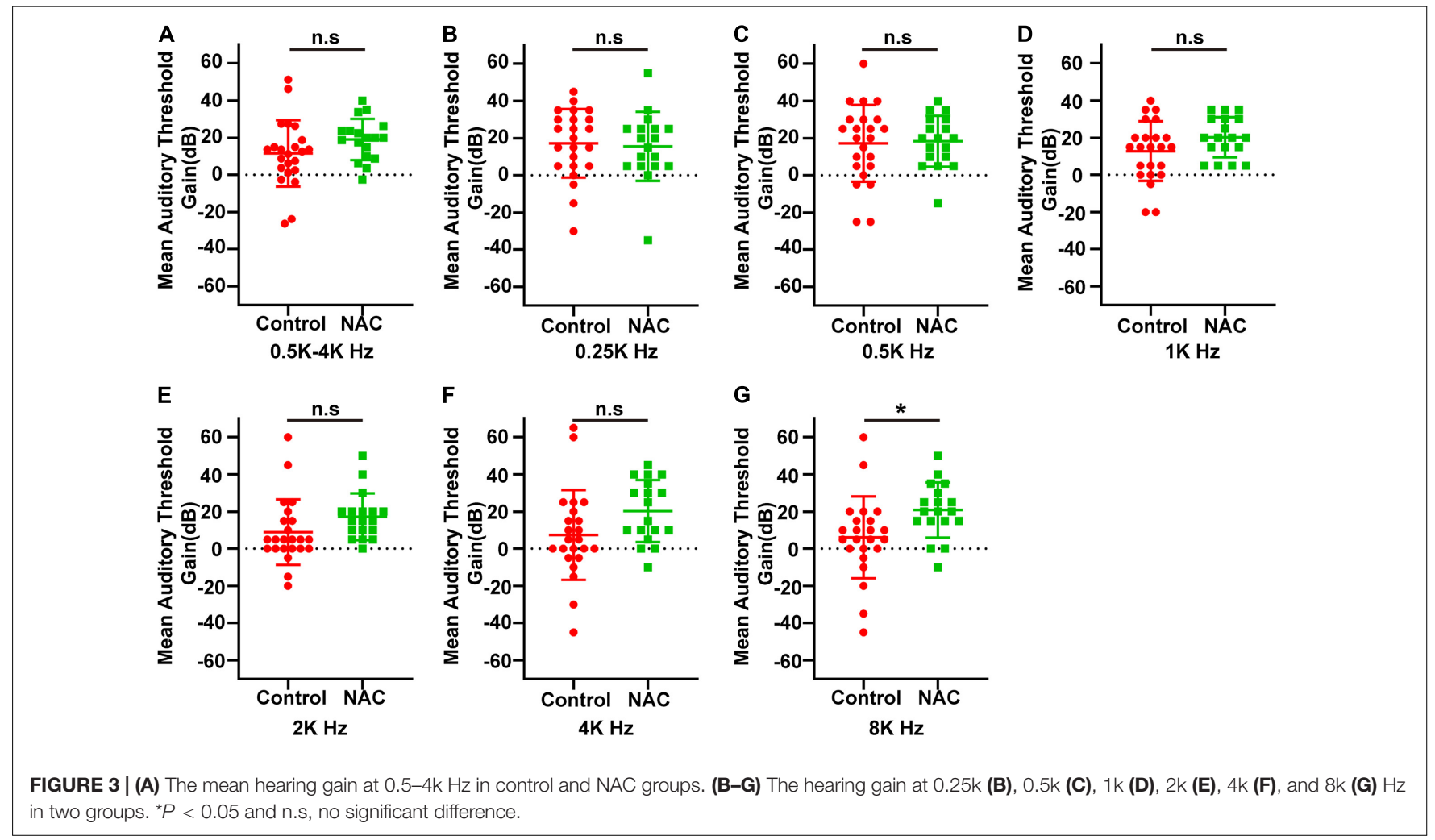

aging, chronic cochlear infections, ototoxic drugs, and noise exposure (Zhu et al., 2018; Zhang Y. et al., 2019; Zhou et al., 2020). The reported mechanisms of hair cell damage mainly include mechanical shearing forces and oxidative damage to HCs (Liu et al., 2016; He et al., 2017; Li et al., 2018; Zhong et al., 2020), eventually induce apoptotic cell death in HCs, especially the outer HCs of the basal turn. The loss of sensory hair cells is irreversible in adult mammals. Although the neonatal cochlea has very limited hair cell regeneration ability, this regeneration ability is rapidly reduced with age (Wang et al., 2015; Zhang S. et al., 2019, 2020; Zhang Y. et al., 2020). It is still a controversy that the mechanism of SSNHL in the cochlea, Capaccio et al. (2012) had done a research indicating that the patients in SSNHL group had higher serum levels of
ROS than in control group, therefore, researcher speculated that hearing loss in SSNHL may be due to antioxidant system failing to handle a sudden rise in ROS. There were studies indicating that excessive ROS, which was produced by noxious stimulation (such as noise, drug) in the cochlea, can destroy hair cell components by oxidizing molecules, such as DNA, proteins (Fechter, 2005; Li and Steyger, 2009). And the unbalance of antioxidant system can activate the programmed cell death pathway in cochlea, causing sensorineural hearing loss (Liu et al., 1998). Therefore, the GO model, a classical oxidative stress model, was used to set up a cochlear explant model of oxidative stress injury for studying SSNHL in vitro in our study. Our data show that NAC and dexamethasone have an obvious synergistic effect in the treatment of hair cell damage 
TABLE 2 | Hearing improvements, respectively, in control and NAC group.

\begin{tabular}{lcc}
\hline & Control group $(\boldsymbol{n}=\mathbf{2 3})$ & NAC group $(\boldsymbol{n}=\mathbf{1 8})$ \\
\hline Hearing improvement (\%) & $9(39 \%)$ & $13(72 \%)$ \\
No improvement (\%) & $14(61 \%)$ & $5(28 \%)$ \\
\hline
\end{tabular}

$P=0.035$.

induced by oxidative stress in vitro. There is plenty of evidence to suggest that NAC used as an antioxidant can attenuate hair cell degeneration or deafness in vitro or in different animal models of deafness (Kopke et al., 2000; Ohinata et al., 2003; Duan et al., 2004; Wang W. et al., 2017; Liu W. et al., 2019). Moreover, glucocorticoids have strong anti-inflammatory, antitoxic immunoregulatory effects. Some studies have reported that glucocorticoids can protect hair cells from a variety of adverse factors, such as noise and inflammation (Hirose et al., 2007; Haake et al., 2015; Müller et al., 2017). Evidence from animal and in vitro experiments suggests that hair cell protection may be one of the common therapeutic effects of NAC and glucocorticoid. In our in vitro experiments, the combination of NAC and glucocorticoids was effective in protecting hair cells, although they were administered at concentrations which were ineffective when used alone. Previous research suggests that NAC may be a steroid sensitizer which can help to treat steroid-resistant asthma in mice (Eftekhari et al., 2013). At present, we do not know whether the protection of hair cells is caused by NAC increasing the therapeutic effect of dexamethasone. However, this interesting finding has potential value for clinical application. It may help us overcome steroid-resistant SSNHL or achieve better results with smaller doses of drugs.

$\mathrm{N}$-acetylcysteine combined with ITD therapy can significantly improve hearing loss at high frequency in SSHNL patients. A study by Machado et al. showed that ITD combined with oral prednisone and NAC can improve hearing loss at $4,000 \mathrm{~Hz}$ as initial therapy. However, it was not until 6 months later that there was a statistically-significant difference between the steroid alone group and the steroids + NAC group (Angeli et al., 2012). In our study, the onset of hearing loss of all patients was at more than 2 weeks. Therefore, a salvage therapy of ITD was adopted without oral steroids. Considering the safety of the drug, the oral dose of NAC was $600 \mathrm{mg}$ two times daily in our design, which was half of the dose used in the study by Machado et al. Since we observed the protection of hair cells in the high frequency region in vitro, all the patients chosen in this study suffered hearing loss in the high frequency region $(4,000$ or $8,000 \mathrm{~Hz})$. Our data showed that ITD combined with NAC as a salvage approach was found to significantly improve high-frequency hearing loss in patients with SSHNL.

The protective effect at high frequency may be caused by increasing the sensitivity of the inner ear to dexamethasone through oral NAC. When ITD is used in clinical practice, both injection dose and interval may affect the efficacy (Liebau et al., 2016). Recent studies found that the therapeutic effect of steroids on SSNHL can be significantly improved through use of a microcatheter with an electronic pump, near-continual transtympanic steroid perfusion or ITD administration using saturated Gelfoam (Chou et al., 2013; Li et al., 2013; Lundy et al., 2018). The above evidence suggested that increasing the amount and the effective time of steroids in the inner ear can significantly improve the therapeutic effect on SSNHL. However, the above method requires a complicated operation and corresponding equipment, making it difficult to popularize at present. Our finding, from another perspective, can achieve similar goals. We speculate that oral NAC may increase the effectiveness of steroids by reducing the minimum effective concentration or extending the effective duration. However, NAC may also help the hearing recovery of SSNHL patients through direct antioxidant effects.

Although there were some interesting findings, our study does have some limitations. Firstly, significant hearing improvement occurred only at high frequency. We and Machado et al. failed to observe any effect of this combination therapy on hearing improvement at low frequencies. Different doses of NAC and different methods of administration may need to be tried in the future. Secondly, the specific therapeutic mechanism of this combined therapy remains unknown. Although one study suggested that NAC alone can improve SSNHL, our in vitro experiment indicated that the synergistic effect of NAC and steroids may play a key role in protecting hair cells from oxidative stress. More evidence is needed to determine whether antioxidant therapy alone is effective against SSHNL. Finally, in our clinical trial, it was difficult to perform subgroup analysis of the different deafness types due to the limited numbers of patients. More extensive studies related to the associations between NAC and subtypes of SSNHL will be conducted with larger numbers of patients in future.

\section{CONCLUSION}

In this study, we found that NAC combined with dexamethasone may protect against hearing damage by protecting hair cells. The results of our clinical study suggest that the use of NAC in combination with ITD is beneficial in the $8,000 \mathrm{~Hz}$ frequency. Combined therapy of NAC and ITD can improve the hearing recovery rate of patients with SSNHL.

\section{DATA AVAILABILITY STATEMENT}

The raw data supporting the conclusions of this article will be made available by the authors, without undue reservation.

\section{ETHICS STATEMENT}

The studies involving human participants were reviewed and approved by the institutional review board of Wuhan Union hospital. Written informed consent to participate in this study was provided by the participants' legal guardian/next of kin. The animal study was reviewed and approved by the institutional 
review board of Wuhan Union hospital. Written informed consent was obtained from the owners for the participation of their animals in this study.

\section{AUTHOR CONTRIBUTIONS}

YS and SC conceived and designed the experiments. XB and KX performed the experiments in vitro. $\mathrm{XB}$ and $\mathrm{XN}$ collect the clinic data. YJ, LX, and YQ analyzed the data. XB, SC, and YS wrote the

\section{REFERENCES}

Alexander, T. H., and Harris, J. P. (2013). Incidence of sudden sensorineural hearing loss. Otol. Neurotol. 34:1586. doi: 10.1097/MAO.0000000000000222

Angeli, S. I., Abi-Hachem, R. N., Vivero, R. J., Telischi, F. T., and Machado, J. J. L.-N. (2012). Acetylcysteine treatment is associated with improved hearing outcome in sudden idiopathic sensorineural hearing loss. Acta Otolaryngol. 132, 369-376. doi: 10.3109/00016489.2011.647359

Becatti, M., Marcucci, R., Mannucci, A., Gori, A. M., Giusti, B., Sofi, F., et al. (2017). Erythrocyte membrane fluidity alterations in sudden sensorineural hearing loss patients: the role of oxidative stress. Thromb. Haemost. 117, 2334-2345. doi: 10.1160/TH17-05-0356

Berjis, N., Soheilipour, S., Musavi, A., and Hashemi, S. M. (2016). Intratympanic dexamethasone injection vs methylprednisolone for the treatment of refractory sudden sensorineural hearing loss. Adv. Biomed. Res. 5:111. doi: 10.4103/22779175.184277

Capaccio, P., Pignataro, L., Gaini, L. M., Sigismund, P. E., Novembrino, C., De Giuseppe, R., et al. (2012). Unbalanced oxidative status in idiopathic sudden sensorineural hearing loss. Eur. Arch. Otorhinolaryngol. 269, 449-453. doi: 10. 1007/s00405-011-1671-2

Chandrasekhar, S. S., Tsai Do, B. S., Schwartz, S. R., Bontempo, L. J., Faucett, E. A., Finestone, S. A., et al. (2019). Clinical practice guideline: sudden hearing loss (Update) executive summary. Otolaryngol. Head Neck Surg. 161, 195-210. doi: $10.1177 / 0194599819859883$

Chen, C. H., and Young, Y. H. (2016). N-acetylcysteine as a single therapy for sudden deafness. Acta Otolaryngol. 137, 58-62. doi: 10.1080/00016489.2016. 1214981

Chou, Y. F., Chen, P. R., Kuo, I. J., Yu, S. H., Wen, Y. H., and Wu, H. P. (2013). Comparison of intermittent intratympanic steroid injection and near-continual transtympanic steroid perfusion as salvage treatments for sudden sensorineural hearing loss. Laryngoscope 123, 2264-2269. doi: 10.1002/lary.23909

Duan, M., Qiu, J., Laurell, G. R., Olofsson, k, Counter, S. A., and Borg, E. (2004). Dose and time-dependent protection of the antioxidant N-L-acetylcysteine against impulse noise trauma. Hear. Res. 192, 1-9. doi: 10.1016/j.heares.2004. 02.005

Eftekhari, P., Hajizadeh, S., Raoufy, M. R., Masjedi, M. R., and Foster, P. S. (2013). Preventive effect of $\mathrm{N}$-acetylcysteine in a mouse model of steroid resistant acute exacerbation of asthma. EXCLI J. 12, 184-192.

Fechter, L. D. (2005). Oxidative stress: a potential basis for potentiation of noiseinduced hearing loss. Environ. Toxicol. Pharmacol. 19, 543-546. doi: 10.1016/j. etap.2004.12.017

Ferri, E., Frisina, A., Fasson, A. C., Armato, E., Spinato, G., and Amadori, M. (2012). Intratympanic steroid treatment for idiopathic sudden sensorineural hearing loss after failure of intravenous therapy. ISRN Otolaryngol. 2012:647271. doi: 10.5402/2012/647271

Haake, S. M., Dinh, C. T., Chen, S., Eshraghi, A. A., and Water, T. R. V. D. (2015). Dexamethasone protects auditory hair cells against TNFalpha-initiated apoptosis via activation of PI3K/Akt and NFkappaB signaling. Hear. Res. 255, 22-32. doi: 10.1016/j.heares.2009.05.003

Hatano, M., Uramoto, N., Okabe, Y., Furukawa, M., and Makoto, I. (2008). Vitamin $\mathrm{E}$ and vitamin $\mathrm{C}$ in the treatment of idiopathic sudden sensorineural hearing loss. Acta Otolaryngol. 128, 116-121. doi: 10.1080/00016480701387132 manuscript. All authors contributed to the article and approved the submitted version.

\section{FUNDING}

The work was supported by grants from the National Nature Science Foundation of China (82071058, 81771003, and 81500793).

He, Z., Guo, L., Shu, Y., Fang, Q., Zhou, H., Liu, Y., et al. (2017). Autophagy protects auditory hair cells against neomycin-induced damage. Autophagy 13, 1884-1904. doi: 10.1080/15548627.2017.1359449

Hirose, Y., Tabuchi, K., Oikawa, K., Murashita, H., and Hara, A. (2007). The effects of the glucocorticoid receptor antagonist RU486 and phospholipase A2 inhibitor quinacrine on acoustic injury of the mouse cochlea. Neurosci. Lett. 413, 63-67. doi: 10.1016/j.neulet.2006.11.029

Hunchaisri, N., Chantapant, S., and Srinangyam, N. (2010). Intratympanic dexamethasone for refractory sudden sensorineural hearing loss. Chotmaihet Thangphaet 93, 1406-1414.

Jarosław, P., Sutkowy, P., Piechocki, J., and Woźniak, A. (2019). Markers of oxidant-antioxidant equilibrium in patients with sudden sensorineural hearing loss treated with hyperbaric oxygen therapy. Oxid. Med. Cell. Longev. 2019:8472346. doi: 10.1155/2019/8472346

Joachims, H. Z., Segal, J., Golz, A., Netzer, A., and Goldenberg, D. (2003). Antioxidants in treatment of idiopathic sudden hearing loss. Otol. Neurotol. 24, 572-575. doi: 10.1097/00129492-200307000-00007

Kang, H. S., Park, J. J., Ahn, S. K., Hur, D. G., and Kim, H. Y. (2013). Effect of high dose intravenous vitamin $\mathrm{C}$ on idiopathic sudden sensorineural hearing loss: a prospective single-blind randomized controlled trial. Eur. Arch. Oto Rhino Laryngol. 270, 2631-2636. doi: 10.1007/s00405-012-2294-y

Kaya, H., Koç, A. K., Sayın, Ý, Güneş, S., Altıntaş, A., Yeğin, Y., et al. (2015). Vitamins A, C, and E and selenium in the treatment of idiopathic sudden sensorineural hearing loss. Eur. Arch. Otorhinolaryngol. 272, 1119-1125. doi: 10.1007/s00405-014-2922-9

Kitoh, R., Nishio, S. Y., and Usami, S. I. (2020). Treatment algorithm for idiopathic sudden sensorineural hearing loss based on epidemiologic surveys of a large Japanese cohort. Acta Otolaryngol. 140, 32-39. doi: 10.1080/00016489.2019. 1687936

Kopke, R. D., Weisskopf, P. A., Boone, J. L., Jackson, R. L., Wester, D. C., Hoffer, M. E., et al. (2000). Reduction of noise-induced hearing loss using L-NAC and salicylate in the chinchilla. Hear. Res. 149, 138-146. doi: 10.1016/S03785955(00)00176-3

Li, H., Feng, G., Wang, H., and Feng, Y. (2015). Intratympanic steroid therapy as a salvage treatment for sudden sensorineural hearing loss after failure of conventional therapy: a meta-analysis of randomized. controlled trials. Clin. Ther. 37, 178-187. doi: 10.1016/j.clinthera.2014.11.009

Li, H., Song, Y., He, Z., Chen, X., Wu, X., Li, X., et al. (2018). Meclofenamic acid reduces reactive oxygen species accumulation and apoptosis, inhibits excessive autophagy, and protects hair cell-like HEI-OC1 cells from cisplatin-induced damage. Front. Cell. Neurosci. 12:139. doi: 10.3389/fncel.2018.00139

Li, H., and Steyger, P. S. (2009). Synergistic ototoxicity due to noise exposure and aminoglycoside antibiotics. Noise Health 11, 26-32. doi: 10.4103/1463-1741. 45310

Li, L., Ren, J., Yin, T., and Liu, W. (2013). Intratympanic dexamethasone perfusion versus injection for treatment of refractory sudden sensorineural hearing loss. Eur. Arch. Oto Rhino Laryngol. 270, 861-867. doi: 10.1007/s00405-0122061-0

Liebau, A., Pogorzelski, O., Salt, A. N., and Plontke, S. K. (2016). Hearing changes after intratympanically applied steroids for primary therapy of sudden hearing loss: a meta-analysis using mathematical simulations of drug delivery protocols. Otol. Neurotol. 38, 19-30. doi: 10.1097/MAO.0000000000001254 
Liu, L., Chen, Y., Qi, J., Zhang, Y., He, Y., Ni, W., et al. (2016). Wnt activation protects against neomycin-induced hair cell damage in the mouse cochlea. Cell Death Dis. 7:e2136. doi: 10.1038/cddis.2016.35

Liu, W., Staecker, H., Stupak, H., Malgrange, B., Lefebvre, P., and Van De Water, T. R. (1998). Caspase inhibitors prevent cisplatin-induced apoptosis of auditory sensory cells. . NeuroReport 9, 2609-2614. doi: 10.1097/00001756-19980803000034

Liu, W., Xu, X., Fan, Z., Sun, G., Han, Y., Zhang, D., et al. (2019). Wnt signaling activates TP53-Induced glycolysis and apoptosis regulator and protects against cisplatin-induced spiral ganglion neuron damage in the mouse cochlea. Antioxid. Redox Signal. 30, 1389-1410. doi: 10.1089/ars.2017.7288

Liu, Y., Qi, J., Chen, X., Tang, M., Chu, C., Zhu, W., et al. (2019). Critical role of spectrin in hearing development and deafness. Sci. Adv. 5:eaav7803. doi: 10.1126/sciadv.aav7803

Lundy, L., Karatayli Ozgursoy, S., and Kleindienst, S. (2018). Intratympanic dexamethasone via saturated gelfoam for idiopathic sudden sensorineural hearing loss. Otolaryngol. Head Neck Surg. 160, 361-363. doi: 10.1177/ 0194599818816306

Michel, O. (2011). The revised version of the german guidelines "sudden idiopathic sensorineural hearing loss". Laryngo Rhino Otologie 90, 290-293. doi: 10.1055/ s-0031-1273721

Moon, I. S., Lee, J. D., Kim, J., Hong, S., and Lee, W. (2011). Intratympanic dexamethasone is an effective method as a salvage treatment in refractory sudden hearing loss. Otol. Neurotol. 32, 1432-1436. doi: 10.1097/MAO. 0b013e318238fc43

Müller, M., Tisch, M., Maier, H., and Lwenheim, H. (2017). Reduction of permanent hearingloss by local glucocorticoid application : guinea pigs with acute acoustic trauma. HNO 65, 1-10. doi: 10.1007/s00106-016-0266-z

Nakashima, T., Sato, H., Gyo, K., Hato, N., Yoshida, T., Shimono, M., et al. (2014). Idiopathic sudden sensorineural hearing loss in Japan. Acta Otolaryngol. 134, 1158-1163. doi: 10.3109/00016489.2014.919406

Niran, H., Chantapant, S., and Sirirattanapan, J. (2015). Effectiveness of oral zinc supplementation in the treatment of idiopathic sudden sensorineural hearing loss (ISSNHL). J. Med. Assoc. Thai. 98, 400-407.

Ohinata, Y., Miller, J. M., and Schacht, J. (2003). Protection from noise-induced lipid peroxidation and hair cell loss in the cochlea. Brain Res. 966, 265-273. doi: 10.1016/S0006-8993(02)04205-1

Ozdamar, K., Sen, A., and Gonel, A. (2019). Assessment of oxidative stress in patients with sudden hearing loss: a non-randomized prospective clinical study. Indian J. Otolaryngol. Head Neck Surg. 71(Suppl 2), 1543-1548. doi: 10.1007/ s12070-019-01623-z

Pathak, S., Stern, C., and Vambutas, A. N. - (2015). Acetylcysteine attenuates tumor necrosis factor alpha levels in autoimmune inner ear disease patients. Immunol. Res. 63, 236-245. doi: 10.1007/s12026-015-8696-3

Qi, J., Liu, Y., Chu, C., Chen, X., Zhu, W., Shu, Y., et al. (2019). A cytoskeleton structure revealed by super-resolution fluorescence imaging in inner ear hair cells. Cell Discov. 5:12. doi: 10.1038/s41421-018-0076-4

Riga, M. G., Chelis, L., Kakolyris, S., Papadopoulos, S., and Danielides, V. (2013). Transtympanic injections of $\mathrm{N}$-acetylcysteine for the prevention of cisplatininduced ototoxicity: a feasible method with promising efficacy. Am. J. Clin. Oncol. 36, 1-6. doi: 10.1097/COC.0b013e31822e006d

Sun, H., Qiu, X., Hu, J., and Ma, Z. (2018). Comparison of intratympanic dexamethasone therapy and hyperbaric oxygen therapy for the salvage treatment of refractory high-frequency sudden sensorineural hearing loss. Am. J. Otolaryngol. 39, 531-535. doi: 10.1016/j.amjoto.2018.06.004

Tillinger, J. A., Gupta, C., Ila, K., Ahmed, J., Mittal, J., Van De Water, T. R., et al. (2018). l-N-acetylcysteine protects outer hair cells against TNFalpha initiated ototoxicity in vitro. Acta Otolaryngol. 138, 676-684. doi: 10.1080/00016489. 2018.1440086

Tong, B., Wang, Q., Dai, Q., Hellstrom, S., and Duan, M. (2020). Efficacy of various corticosteroid treatment modalities for the initial treatment of idiopathic sudden hearing loss: a prospective randomized controlled trial. Audiol. Neurootol. 26, 45-52. doi: 10.1159/000508124

Wang, T., Chai, R., Kim, G. S., Pham, N., Jansson, L., Nguyen, D. H., et al. (2015). Lgr5+ cells regenerate hair cells via proliferation and direct transdifferentiation in damaged neonatal mouse utricle. Nat. Commun. 6:6613. doi: 10.1038/ ncomms7613

Wang, W., Li, D., Ding, X., Zhao, Q., Chen, J., Tian, K., et al. (2017). NAcetylcysteine protects inner ear hair cells and spiral ganglion neurons from manganese exposure by regulating ROS levels. Toxicol. Lett. 279, 77-86. . . doi: 10.1016/j.toxlet.2017.07.903

Wang, Y., Li, J., Yao, X., Li, W., Du, H., Tang, M., et al. (2017). Loss of CIB2 causes profound hearing loss and abolishes mechanoelectrical transduction in mice. Front. Mol. Neurosci. 10:401. doi: 10.3389/fnmol.2017.00401

Yang, C. H., Ko, M. T., Peng, J. P., and Hwang, C. F. (2011). Zinc in the treatment of idiopathic sudden sensorineural hearing loss. Laryngoscope 121, 617-621. doi: 10.1002/lary.21291

Zhang, S., Liu, D., Dong, Y., Zhang, Z., Zhang, Y., Zhou, H., et al. (2019). Frizzled$9+$ supporting cells are progenitors for the generation of hair cells in the postnatal mouse cochlea. Front. Mol. Neurosci. 12:184. doi: 10.3389/fnmol.2019. 00184

Zhang, S., Qiang, R., Dong, Y., Zhang, Y., Chen, Y., Zhou, H., et al. (2020). Hair cell regeneration from inner ear progenitors in the mammalian cochlea. Am. J. Stem Cells 9, 25-35.

Zhang, Y., Li, W., He, Z., Wang, Y., Shao, B., Cheng, C., et al. (2019). Pre-treatment with fasudil prevents neomycin-induced hair cell damage by reducing the accumulation of reactive oxygen species. Front. Mol. Neurosci. 12:264. doi: 10.3389/fnmol.2019.00264

Zhang, Y., Zhang, S., Zhang, Z., Dong, Y., Ma, X., Qiang, R., et al. (2020). Knockdown of Foxg1 in Sox9+ supporting cells increases the transdifferentiation of supporting cells into hair cells in the neonatal mouse utricle. Aging 12, 19834-19851. doi: 10.18632/aging.104009

Zhong, Z., Fu, X., Li, H., Chen, J., Wang, M., Gao, S., et al. (2020). Citicoline protects auditory hair cells against neomycin-induced damage. Front. Cell Dev. Biol. 8:712. doi: 10.3389/fcell.2020.00712

Zhou, H., Qian, X., Xu, N., Zhang, S., Zhu, G., Zhang, Y., et al. (2020). Disruption of Atg7-dependent autophagy causes electromotility disturbances, outer hair cell loss, and deafness in mice. Cell Death Dis. 11:913. doi: 10.1038/s41419-02003110-8

Zhu, C., Cheng, C., Wang, Y., Muhammad, W., Liu, S., Zhu, W., et al. (2018). Loss of ARHGEF6 causes hair cell stereocilia deficits and hearing loss in mice. Front. Mol. Neurosci. 11:362. doi: 10.3389/fnmol.2018.00362

Conflict of Interest: The authors declare that the research was conducted in the absence of any commercial or financial relationships that could be construed as a potential conflict of interest.

Copyright (C) 2021 Bai, Chen, Xu, Jin, Niu, Xie, Qiu, Liu and Sun. This is an openaccess article distributed under the terms of the Creative Commons Attribution License (CC BY). The use, distribution or reproduction in other forums is permitted, provided the original author(s) and the copyright owner(s) are credited and that the original publication in this journal is cited, in accordance with accepted academic practice. No use, distribution or reproduction is permitted which does not comply with these terms. 